\title{
Transfection of S2 Cell with DNA Using CellFectin Reagent
}

Darran G. Cronshaw*

Center for Autoimmune and Musculoskeletal Diseases, Feinstein Institute for Medical Research, Manhasset, USA

*For correspondence: dcronshaw@nshs.edu

[Abstract] This method provides a step-by-step guide to transfecting Drosophila S2 cells with the pRmHA-3 (or similar) vector with insert of choice (in this case SDF-1 $\beta-F L A G$ ) and generating a stable cell line. This cell line is then capable of producing the protein of interest under inducible conditions by addition of copper sulfate, which can then be purified and used as desired. This protocol provides an example to finding out when your peak protein production occurs and a method for determining optimal selection conditions.

\section{Materials and Reagents}

1. Drosophila Schneider 2 (S2) cells (Life Technologies, Invitrogen ${ }^{\mathrm{TM}}$, catalog number: R690-07)

2. Dros-SFM (Life Technologies, Invitrogen ${ }^{\mathrm{TM}}$, catalog number: 10797-025)

3. pHAS $\beta A G$ (pRmHA-3 vector with SDF-1 $\beta$-FLAG inserted. Contains a metallothionein promoter inducible with $\mathrm{Cu}^{2+}$ )

4. pUC-Hsneo (contains the neomycin-resistant cassette required for stable selection)

5. CellFectin reagent (Life Technologies, Invitrogen ${ }^{\mathrm{TM}}$, catalog number: 10362-010)

6. FBS (Life Technologies, Invitrogen ${ }^{\mathrm{TM}}$, catalog number: 10437-028)

7. Penicillin-Streptomycin (P/S) (Life Technologies, Invitrogen ${ }^{\mathrm{TM}}$, catalog number: 15140122)

8. G418 sulphate (Life Technologies, Invitrogen ${ }^{\mathrm{TM}}$, catalog number: 11811-031)

9. $\mathrm{CuSO}_{4}$ (Sigma Aldrich, catalog number: C8027)

10. HT supplement (Life Technologies, Invitrogen ${ }^{\text {TM }}$, catalog number: 11067-030)

11. Transfection solutions (see Recipes)

\section{Equipment}

1. 6-well plate (Corning Incorporated, catalog number: 353046)

2. 24-well plate (Corning Incorporated, catalog number: 353047) 


\section{Procedure}

1. Drosophila Schneider 2 (S2) cells are plated out at $1.6 \times 10^{6}$ cells/well (6-well plate, $2 \mathrm{ml}$ /well) and incubated overnight at room temperature (RT).

Note: The optimal temperature of $\mathrm{S} 2$ cells is actually $28{ }^{\circ} \mathrm{C}$ and they do not need $\mathrm{CO}_{2}$ to culture.

2. Following day, cells are washed $2 x$ with Dros-SFM (no FBS, P/S or HT) 1 ml/well.

3. Prepare the 'DNA mix' and 'CellFectin Mix' transfection solutions.

\begin{tabular}{|c|c|c|c|}
\hline 6-well plate & $\begin{array}{l}0.5 \mathrm{mg} / \mathrm{ml} \\
\mathrm{G} 418 \\
\text { Un-transfected }\end{array}$ & $\begin{array}{l}1.0 \mathrm{mg} / \mathrm{ml} \\
\mathrm{G} 418 \\
\text { Untransfected }\end{array}$ & $\begin{array}{l}1.5 \mathrm{mg} / \mathrm{ml} \\
\mathrm{G} 418 \\
\text { Untransfected }\end{array}$ \\
\hline & $\begin{array}{l}\text { No G418 } \\
\text { Un-transfected }\end{array}$ & $\begin{array}{l}\text { No G418 } \\
\text { Transfected w. } \\
4 \text { ul CelliFectin }\end{array}$ & $\begin{array}{l}\text { No G418 } \\
\text { Transfected w. } \\
8 \text { ㅆl CelliFectin }\end{array}$ \\
\hline
\end{tabular}

\begin{tabular}{|c|c|c|}
\hline $\begin{array}{l}0.5 \mathrm{mg} / \mathrm{ml} \\
\mathrm{G} 418\end{array}$ & $\begin{array}{l}1.0 \mathrm{mg} / \mathrm{ml} \\
\mathrm{G} 418\end{array}$ & $\begin{array}{l}1.5 \mathrm{mg} / \mathrm{ml} \\
\mathrm{G} 418\end{array}$ \\
\hline $\begin{array}{l}\text { Transfected } w \text {. } \\
4 \mu \mathrm{l} \text { CellFectin }\end{array}$ & $\begin{array}{l}\text { Transfected } w \text {. } \\
4 \mu \mathrm{l} \text { CellFectin }\end{array}$ & $\begin{array}{l}\text { Transfected } w \text {. } \\
4 \mu \text { l CellFectin }\end{array}$ \\
\hline $\begin{array}{l}0.5 \mathrm{mg} / \mathrm{ml} \\
\mathrm{G} 418\end{array}$ & $\begin{array}{l}1.0 \mathrm{mg} / \mathrm{ml} \\
\mathrm{G} 418\end{array}$ & $\begin{array}{l}1.5 \mathrm{mg} / \mathrm{ml} \\
\mathrm{G} 418\end{array}$ \\
\hline $\begin{array}{l}\text { Transfected w. } \\
8 \mu \mathrm{l} \text { CellFectin }\end{array}$ & $\begin{array}{l}\text { Transfected } w \text {. } \\
8 \mu \mathrm{l} \text { CellFectin }\end{array}$ & $\begin{array}{l}\text { Transfected } w \text {. } \\
8 \mu \mathrm{l} \text { CellFectin }\end{array}$ \\
\hline
\end{tabular}

4. $100 \mu \mathrm{l}$ /sample (i.e. $400 \mu \mathrm{l}$ total) of the DNA mix is added to $100 \mu \mathrm{l} /$ sample (i.e. $400 \mu \mathrm{l}$ total) CellFectin mix. Solution is gently mixed and left at RT for $30 \mathrm{~min}$.

5. After $30 \mathrm{~min}, 800 \mu \mathrm{l} /$ sample ( $3.2 \mathrm{ml}$ total) of Dros-SFM (no FBS, P/S or HT) is added to the DNA/CellFectin mix.

6. $1 \mathrm{ml}$ of this solution is then added to the appropriate wells containing the $\mathrm{S} 2$ cells and incubated at RT for $5 \mathrm{~h}$.

7. Following the $5 \mathrm{~h}$ incubation, medium is aspirated off and Dros-SFM $+10 \% \mathrm{FBS}+\mathrm{P} / \mathrm{S}+$ $\mathrm{HT}$ (= complete media) is added to each well $(2 \mathrm{ml} / \mathrm{well})$.

8. Plates are incubated at RT for $48 \mathrm{~h}$.

9. At $48 \mathrm{~h}$ post-transfection the media is removed and replaced with $2 \mathrm{ml}$ media containing G418 sulphate at indicated concentrations. The cells are transferred to 24-well plates (one 6-well $\geq$ eight 24-wells) supplemented with additional media + G418 to $1 \mathrm{ml}$.

\begin{tabular}{|c|c|c|c|}
\hline \multirow{3}{*}{$\begin{array}{l}\text { 24-well plate } \\
\text { layout }\end{array}$} & $\begin{array}{l}\text { No G418 } \\
\text { Un-transfected }\end{array}$ & $\begin{array}{l}\text { No G418 } \\
\text { Transfected w. } \\
4 \mu \mathrm{l} \text { CellFectin }\end{array}$ & $\begin{array}{l}\text { No G418 } \\
\text { Transfected w. } \\
8 \mu \text { l CellFectin }\end{array}$ \\
\hline & $\begin{array}{l}0.5 \mathrm{mg} / \mathrm{ml} \\
\mathrm{G} 418\end{array}$ & $\begin{array}{l}1.0 \mathrm{mg} / \mathrm{ml} \\
\mathrm{G} 418\end{array}$ & $\begin{array}{l}1.5 \mathrm{mg} / \mathrm{ml} \\
\mathrm{G} 418\end{array}$ \\
\hline & $\begin{array}{l}\text { Transfected w. } \\
4 \mu \mathrm{l} \text { CellFectin }\end{array}$ & $\begin{array}{l}\text { Transfected w. } \\
4 \mu \text { l CellFectin }\end{array}$ & $\begin{array}{l}\text { Transfected w. } \\
4 \mu \text { l CellFectin }\end{array}$ \\
\hline
\end{tabular}

\begin{tabular}{|l|l|l|}
\hline $\begin{array}{l}0.5 \mathrm{mg} / \mathrm{ml} \\
\text { G418 }\end{array}$ & $\begin{array}{l}1.0 \mathrm{mg} / \mathrm{ml} \\
\text { G418 }\end{array}$ & $\begin{array}{l}1.5 \mathrm{mg} / \mathrm{ml} \\
\text { G418 }\end{array}$ \\
Un-transfected & Untransfected & Untransfected \\
\hline $\begin{array}{l}0.5 \mathrm{mg} / \mathrm{ml} \\
\mathrm{G} 418\end{array}$ & $\begin{array}{l}1.0 \mathrm{mg} / \mathrm{ml} \\
\mathrm{G} 418\end{array}$ & $\begin{array}{l}1.5 \mathrm{mg} / \mathrm{ml} \\
\mathrm{G} 418\end{array}$ \\
$\begin{array}{l}\text { Transfected } w \\
8 \mu \mathrm{l} \text { CellFectin }\end{array}$ & $\begin{array}{l}\text { Transfected } w \\
8 \mu \mathrm{l} \text { CellFectin }\end{array}$ & $\begin{array}{l}\text { Transfected } w \\
8 \mu \mathrm{l} \text { CellFectin }\end{array}$ \\
\hline
\end{tabular}

10. Cells are incubated at RT with media changed every 5 days $(1 \mathrm{ml} /$ well).

11. After 3 weeks, untransfected cells should be killed by the G418 at least at the highest concentration. The transfected cells should be fine and healthy. 
12. Cells within the $4 \mu \mathrm{l}$ and $8 \mu \mathrm{l}$ CellFectin groups are pooled and split 1:1 into two 24-well plates (i.e. 8 wells /plate). After $1 \mathrm{~h}$, media is removed and replaced with either complete $\mathrm{SFM}+1.5 \mathrm{mg} / \mathrm{ml} \mathrm{G} 418$ or complete SFM $+1.5 \mathrm{mg} / \mathrm{ml} \mathrm{G} 418+1 \mathrm{mM} \mathrm{CuSO}_{4}$.

\begin{tabular}{|c|c|c|c|c|}
\hline & $\begin{array}{l}1.5 \mathrm{mg} / \mathrm{ml} \\
\mathrm{G} 418\end{array}$ & $\begin{array}{l}1.5 \mathrm{mg} / \mathrm{ml} \\
\mathrm{G} 418\end{array}$ & $\begin{array}{l}1.5 \mathrm{mg} / \mathrm{ml} \\
\mathrm{G} 418\end{array}$ & $\begin{array}{l}1.5 \mathrm{mg} / \mathrm{ml} \\
\mathrm{G} 418\end{array}$ \\
\hline layout & $\begin{array}{l}\text { Transfected } \\
\text { w. } 4 \mu \mathrm{l} \\
\text { CellFectin }\end{array}$ & $\begin{array}{l}\text { Transfected } \\
\text { w. } 8 \mu \mathrm{l} \\
\text { CellFectin }\end{array}$ & $\begin{array}{l}\text { Transfected } \\
\text { w. } 4 \mu \mathrm{l} \\
\text { CellFectin }\end{array}$ & $\begin{array}{l}\text { Transfected } \\
\text { w. } 8 \mu \mathrm{l} \\
\text { CellFectin }\end{array}$ \\
\hline
\end{tabular}

13. Cell lysates are taken at 2, 3, 4 and 5 days post-CuSO 4 addition to determine peak time of SDF-1 $\beta$-FLAG production.

\section{$\underline{\text { Recipes }}$}

1. Transfection solutions
a. DNA mix (per sample):
Total (9 samples)
$4 \mu \mathrm{pHAS} \beta A G=1.67 \mu \mathrm{l}$
$15.0 \mu \mathrm{l}$
$0.2 \mu \mathrm{g}$ pUC-Hsneo $=0.63 \mu \mathrm{l}$
$5.7 \mu \mathrm{l}$
$100 \mu$ l Dros-SFM (no FBS or P/S)
$900 \mu \mathrm{l}$
b. CellFectin Mix (per sample):
Total (4 samples)
$4 \mu \mathrm{l}$ or $8 \mu \mathrm{l}$ CellFectin reagent
16 or $32 \mu \mathrm{l}$
$100 \mu \mathrm{l}$ Dros-SFM
$400 \mu \mathrm{l}$

\section{References}

1. Bunch, T. A., Grinblat, Y. and Goldstein, L. S. (1988). Characterization and use of the Drosophila metallothionein promoter in cultured Drosophila melanogaster cells. Nucleic Acids Res 16(3): 1043-1061. 